\title{
VISÃO DOS TÉCNICOS E AUXILIARES DE ENFERMAGEM SOBRE O ESTILO DE LIDERANÇA DO ENFERMEIRO*
}

\author{
Joseane Santos Alecrim ${ }^{1}$, Luciana de Freitas Campos ${ }^{2}$
}

\begin{abstract}
RESUMO: Objetivos: identificar o estilo de liderança dos enfermeiros hospitalares sob a óptica dos auxiliares e técnicos de enfermagem. Investigação exploratória, com abordagem quantitativa realizada em um hospital do interior de Minas Gerais, tendo 44 profissionais de enfermagem de nível médio como sujeitos da pesquisa, com idade média de 34,7 anos cuja maioria é do sexo feminino. Para os auxiliares de enfermagem não foi verificada a diferença entre os estilos de liderança exercidos pelo enfermeiro. Já os técnicos de enfermagem definem os estilos de liderança exercidos pelo enfermeiro: a gerência em equipe e a gerência empobrecida. Espera-se que o enfermeiro atualize constantemente o seu conhecimento acerca da liderança e desenvolva habilidades, neste sentido, no intuito de influenciar a equipe de enfermagem promovendo a motivação humana no desenvolvimento do trabalho.
\end{abstract}

PALAVRAS-CHAVE: Administração hospitalar; Liderança; Enfermagem.

\section{THE VIEW OF NURSING TECHNICIANS AND AUXILIARIES ON THE NURSING LEADERSHIP STYLE}

\begin{abstract}
This study aims to identify and discuss the leadership style of hospital nurses from the perspective of nursing auxiliaries and technicians. An exploratory research with a quantitative approach was carried out at a hospital in the interior of Minas Gerais, involving 44 secondary-level nursing professionals with an average age of 34.7 years and mostly women. Among the nursing auxiliaries, no difference was found between the leadership styles practices by the nurse. The nursing technicians, on the other hand, define the leadership styles practiced by the nurse: in the management time and the poor management. Nurses are expected to constantly update their knowledge about leadership and develop skills, in this sense, with a view to influencing the nursing team, promoting human motivation in work practice.
\end{abstract}

KEYWORDS: Hospital administration; Leadership; Nursing.

\section{VISIÓN DE LOS TÉCNICOS Y AUXILIARES DE ENFERMERÍA SOBRE ELESTILO DE LIDERAZGO DEL ENFERMERO}

RESUMEN: Objetivos: identificar el estilo de liderazgo de los enfermeros hospitalarios bajo la óptica de los auxiliares y técnicos de enfermería. Investigación exploratoria, abordaje cuantitativa realizada en un hospital del interior de Minas Gerais, involucrando 44 profesionales de enfermería de nivel medio como sujetos de la investigación, con edad promedio de 34,7 años cuya mayoría es del sexo femenino. Para los auxiliares de enfermería, no fue verificada la diferencia entre los estilos de liderazgo ejercidos por el enfermero. Ya los técnicos de enfermería definen los estilos de liderazgo ejercidos por el enfermero: la gerencia en equipo y la gerencia empobrecida. Se espera que el enfermero actualice constantemente su conocimiento acerca del liderazgo y desarrolle habilidades, en este sentido, para influenciar el equipo de enfermería, promoviendo la motivación humana en el desarrollo del trabajo.

PALABRAS CLAVE: Administración hospitalaria; Liderazgo; Enfermería.

*Trabalho vinculado ao Grupo de Estudos de Gerenciamento em Serviços de Saúde e Enfermagem-GEGSSE, cadastrado no CNPq. ${ }^{1}$ Discente do Curso de Graduação em Enfermagem da Universidade Federal dos Vales do Jequitinhonha e Mucuri-UFVJM. Estudante pesquisadora do GEGSSE.

${ }^{2}$ Enfermeira. Licenciada em Enfermagem. Especialista em Enfermagem do Trabalho. Mestre em Enfermagem Fundamental. Docente. Orientadora do Trabalho. Líder do GEGSSE.

Autor correspondente:

Luciana de Freitas Campos

Universidade Federal dos Vales do Jequitinhonha e Mucuri

Praça Prof. José Augusto Neves, 43 - 39300-000 - Diamantina-MG, Brasil

Recebido: 24/08/09

E-mail: camposlf@gmail.com

Aprovado: 10/09/09

Cogitare Enferm 2009 Out/Dez; 14(4):628-37 


\section{INTRODUÇÃO}

Os estudos sobre a liderança no Brasil podem ser agrupados em abordagens que tiveram predomínio em momentos distintos. A abordagem que considerou as características do líder, seus traços e qualidades inatas, dominou até o final dos anos 40 do século XX. A perspectiva do estilo de liderança mudou o foco das características do líder para o seu comportamento e, portanto, para a perspectiva de seu treinamento, que teve importância até os anos 60, com ênfase na concepção do trabalho em equipe influenciada pela corrente humanista da Administração. Dos anos 60 ao início dos anos 80 , predominou a abordagem contingencial, que colocou em lugar central os fatores situacionais, do contexto, para compreender a liderança ${ }^{(1)}$.

Segundo uma perspectiva mais atual de liderança, o enfermeiro deve estar orientado para as possibilidades de desempenhar um novo papel de líder, mais orientado para o futuro, mais flexível, dinâmico e disposto a assumir riscos, em contraposição ao papel controlador, ditador de regras, normas e procedimentos ${ }^{(2)}$. Além disso, cabe ao enfermeiro desenvolver características que o tornem um líder, agir com equidade, eficiência e eficácia para implantar novos modelos de atenção e gerenciamento que provoquem mudanças positivas na assistência ${ }^{(3)}$.

Ressalta-se que cada membro da equipe deve ter consciência da relevância de seu trabalho e que o enfermeiro líder saiba evidenciar essa relação. A marca da liderança moderna é o fortalecimento do grupo de trabalho, evidenciando e valorizando as competências individuais, diluindo o poder na equipe ${ }^{(2)}$.

No âmbito conceitual, entende-se por liderança a ação de influenciar pessoas e comportamentos, ligada a um processo de grupo e/ou de equipe em que se tem um objetivo a atingir ${ }^{(4)}$. Nesse sentido, a liderança pode favorecer a criação e implementação de ações alternativas para garantir a eficiência do serviço de enfermagem e, para tal, faz-se necessário que o enfermeiro se atualize e promova além de seu crescimento pessoal e profissional, o da equipe, salientando-se que além do conhecimento prático é importante que o mesmo desenvolva conhecimento teórico como forma de garantir sua capacitação profissional ${ }^{(5)}$.

O cuidar em saúde e enfermagem exige uma formação que privilegie não só o preparo técnicocientífico, mas, também, a consciência da utilização de qualidades humanas, tão valorizada na perspectiva da gestão contemporânea em que o trabalho do enfermeiro líder visa o alcance de objetivos comuns à instituição, às equipes de trabalho e ao cliente. Neste caso, a liderança na enfermagem, como um fenômeno de grupo, será entendida pelo líder como um desafio no sentido de compartilhar idéias, esforços e recursos para satisfação de clientes e profissionais ${ }^{(6)}$.

Um fator marcante que pode influenciar os membros da equipe de enfermagem e, consequentemente, aumentar a qualidade da assistência, além da satisfação profissional, é a motivação, definida como uma inclinação para a ação que tem origem em um motivo, uma necessidade que, ao atuar sobre o intelecto, faz o indivíduo movimentarse ou agir ${ }^{(7)}$. No entanto, o enfermeiro necessita atentar que a motivação existe dentro de cada indivíduo e se constitui um mistério a ser desvendado e conhecido por todos os líderes ${ }^{(8)}$, sendo que a abordagem à subjetividade de cada membro da equipe deve ser adequadamente trabalhada. Assim, ressalta-se que compete ao enfermeiro motivar sua equipe de trabalho, bem como capacitá-la para que realize uma assistência de qualidade aos pacientes.

Diante do exposto, o enfermeiro pode apresentar diversos estilos de liderança frente à equipe de enfermagem. Estilos estes que, da observação empírica da prática por meio de aulas teóricas, teórico-práticas e estágios, despertaram interesse devido à possibilidade de compreender a organização do serviço baseada em alguma teoria de gestão, apreendida da visão dos auxiliares e técnicos de enfermagem. Este estudo segue a perspectiva de contribuir no direcionamento de ações, comportamentos e atitudes que influenciem os líderes e liderados no desenvolvimento da assistência como um objetivo compartilhado.

Assim, por ser esta uma demanda empiricamente observada a ser explorada na realidade vivenciada e pela consequente necessidade de se definir os estilos de liderança exercidos pelos enfermeiros segundo a visão dos auxiliares e técnicos de enfermagem foi utilizada a Teoria do Grid Gerencial, elaborada por Robert Blake e Jane Mouton ${ }^{(11)}$, a partir dos resultados encontrados nos estudos sobre liderança. Esses autores destacaram, nessa teoria, cinco estilos diferentes de liderança, focalizados por meio de duas dimensões básicas: orientação para a produção (relacionado a resultados, desempenho, lucros fundamentados na decisão, nos prazos, na eficiência, na qualidade e eficácia dos serviços) e orientação para as pessoas (referente à conquista de resultados com 
base na confiança, respeito, obediência, simpatia, apoio) e desenvolveram uma rede de combinações possíveis entre elas a autoridade-obediência $(9,1)$; gerência do clube recreativo $(1,9)$; a gerência empobrecida $(1,1)$; a gerência do homem organizacional $(5,5)$ e a gerência em equipe $(9,9)$, exemplificado na figura abaixo ${ }^{(9,10)}$ :

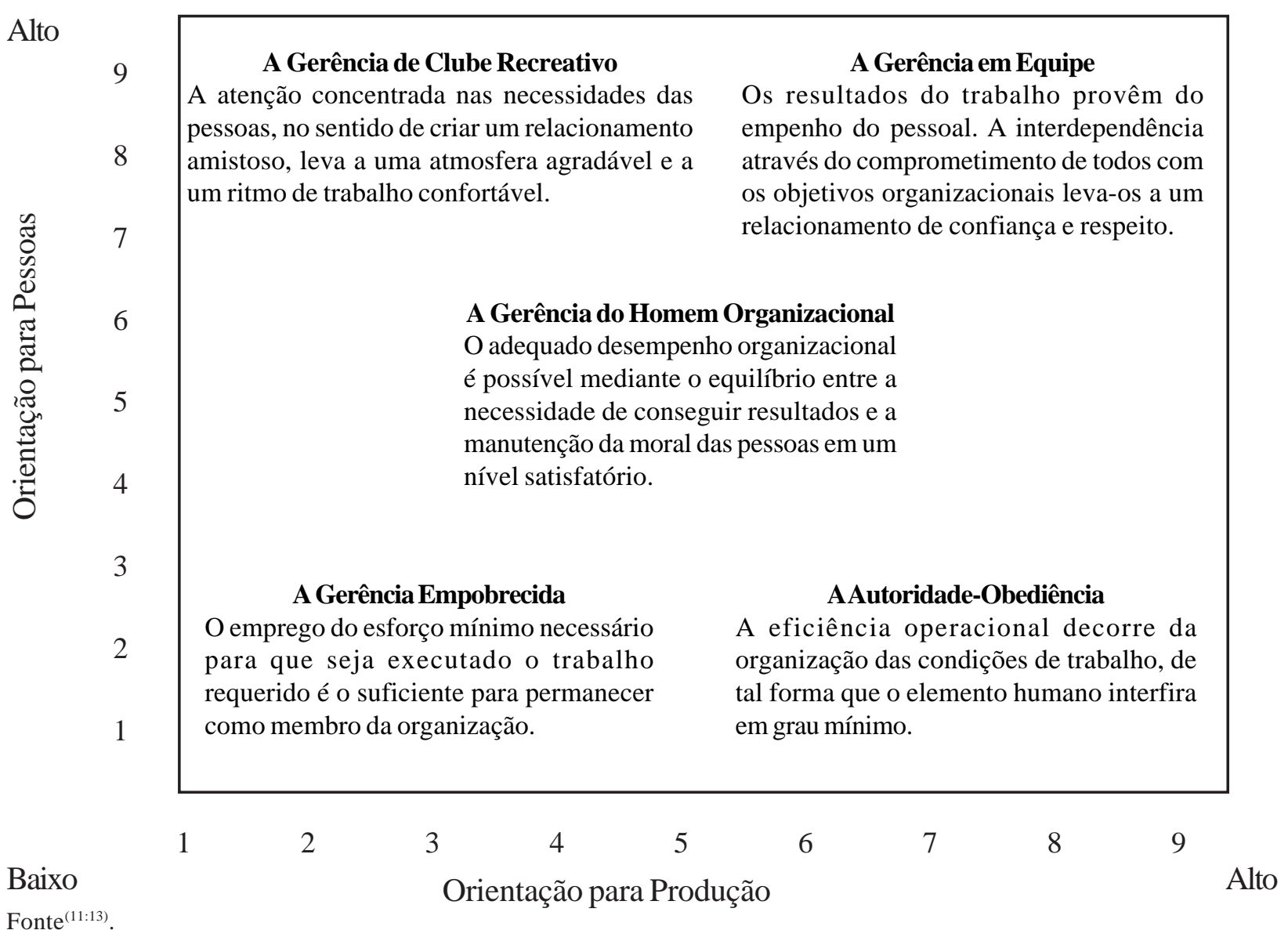

Figura 1- Estilos de liderança segundo a Teoria do Grid Gerencial

O eixo horizontal da Figura 1 representa a orientação para produção e o eixo vertical a orientação para pessoas sendo que em cada um deles apresenta uma indicação do grau de interesse, com uma escala de 9 pontos (1 é o nível mais baixo, 5 o nível médio e 9 o mais alto grau de orientação).

O presente estudo tem por objetivo identificar o estilo de liderança dos enfermeiros hospitalares sob a óptica dos auxiliares e técnicos de enfermagem.

\section{METODOLOGIA}

Trata-se de uma investigação de natureza exploratória, utilizando a abordagem de pesquisa quantitativa, realizada em um hospital filantrópico de médio porte de uma cidade do interior de Minas Gerais. Foram escolhidas a Unidade de Internação de Clínica Médica, a Unidade de Internação da Clínica Cirúrgica, o Centro de Terapia Intensiva-CTI e a Unidade de
Pronto Atendimento-PA.

Os sujeitos da pesquisa foram auxiliares e técnicos de enfermagem, que somados compõem uma amostra de 57 profissionais de nível médio. Destes, 44 concordaram em participar do estudo. Foram excluídos da pesquisa aqueles que discordaram em participar por motivos como: afastamento para férias, licença paternidade, troca de plantão e recusa em responder ao instrumento, totalizando 13 profissionais.

Os dados foram coletados por meio de questionário (Anexo) contendo questões fechadas sobre dados de identificação e dados de proposição que caracterizam o estilo de liderança do enfermeiro, norteadoras do estudo.

O questionário utilizado foi o "Instrumento Grid e Liderança em Enfermagem: Comportamento Real” baseado no estudo de Blake e Mouton ${ }^{(10)}$, por representar um estudo de relevância para esta temática na área de enfermagem. Este instrumento sofreu 
adaptações no item "identificação" quanto à categoria profissional e unidade de internação, devido à característica do local de estudo.

A análise dos questionários foi feita mediante cálculos estatísticos como cálculo de média, análise de variância e Teste de Duncan das 25 proposições respondidas pelos sujeitos da pesquisa, com posterior classificação segundo os estilos de liderança baseados no Grid Gerencial.

O instrumento de coleta de dados foi entregue ao auxiliar ou ao técnico de enfermagem pela pesquisadora, durante o turno de trabalho, nos períodos diurno e noturno, conforme escala mensal de distribuição do pessoal de enfermagem do mês de outubro de 2008, sendo sempre entregue no início do plantão e recolhido ao seu término. O Termo de Consentimento Livre e Esclarecido foi apresentado, explicitado o direito de o sujeito da pesquisa recusar a participar ou se excluir do estudo a qualquer momento se julgar necessário, bem como foi também informado sobre o direito de anonimato e sigilo, sendo as possíveis dúvidas sanadas pela pesquisadora e o termo foi assinado pelos sujeitos em duas vias, permanecendo uma de posse dos mesmos e outra, da pesquisadora.

Foi encaminhado o ofício ao hospital do local de estudo, solicitando autorização para realização da pesquisa. O projeto foi aprovado pelo Comitê de Ética em Pesquisa da Universidade Federal dos Vales do Jequitinhonha e Mucuri, sob o protocolo n ${ }^{\circ} 007 / 08$. A pesquisa apresentou riscos mínimos referentes ao constrangimento por não saber responder alguma questão. O benefício do estudo consiste em promover ou ampliar discussões acerca da liderança em enfermagem, favorecendo a adequação do Modelo de Gestão do Serviço de Enfermagem adotado e consequente ampliação na qualidade de assistência de enfermagem prestada.

\section{RESULTADOS E DISCUSSÃO}

Dos 44 sujeitos da pesquisa, 30 preencheram o quesito idade e apresentaram idade média de 34,7 anos. Quanto à classificação segundo o sexo, 32 profissionais responderam a questão, sendo que da categoria dos auxiliares 15 são do sexo feminino e 1 masculino e dos técnicos de enfermagem, 15 profissionais são do sexo feminino e 1 masculino. Os sujeitos da pesquisa tiveram como referência para responder as proposições o enfermeiro do plantão no qual o questionário foi entregue. Desta forma, os 44 sujeitos o fizeram tomando por base o total de 8 enfermeiros das quatro unidades estudadas.

Quadro 1 - Distribuição da frequência dos 44 sujeitos que responderam sobre o comportamento real dos enfermeiros escalados por unidade de internação. Diamantina, 2008

\begin{tabular}{|c|c|c|c|c|c|c|c|c|c|}
\hline \multirow[t]{2}{*}{ Unidade } & \multicolumn{2}{|c|}{ PA } & \multicolumn{2}{|c|}{ Médica } & \multicolumn{2}{|c|}{ Cirúrgica } & \multicolumn{2}{|c|}{ CTI } & \\
\hline & Enf & (f) & Enf & (f) & Enf & (f) & Enf & (f) & \\
\hline \multirow[t]{3}{*}{ Enfermeiros referenciados } & A & 2 & D & 12 & D & 2 & $\mathrm{~F}$ & 3 & - \\
\hline & B & 2 & $\mathrm{E}$ & 8 & $\mathrm{E}$ & 2 & G & 3 & \\
\hline & $\mathrm{C}$ & 2 & & & & & $\mathrm{H}$ & 8 & \\
\hline Total (enfermeiros) & 3 & - & 2 & - & 2 & - & 3 & - & 8 \\
\hline Total (respondentes) & \multicolumn{2}{|c|}{6} & \multicolumn{2}{|c|}{20} & \multicolumn{2}{|c|}{4} & \multicolumn{2}{|c|}{14} & 44 \\
\hline
\end{tabular}

Fonte: dados da pesquisa de campo

As alternativas de resposta de cada proposição tiveram os seguintes valores: 4 (concordo plenamente); 3 (mais concordo que discordo); 2 (mais discordo que concordo) e 1 (discordo totalmente). As respostas com os valores atribuídos pelos sujeitos da pesquisa foram registradas para cada estilo Grid Gerencial ${ }^{(1)} \mathrm{em}$ um impresso favorecendo a obtenção do total de pontos.

De posse dos dados coletados, os mesmos foram divididos em Grupo 1: Auxiliares $\backslash$ Comportamento e Grupo 2: Técnicos\Comportamento e, então, procedemos para a identificação da liderança esperada para o enfermeiro.

Na Tabela 1, os auxiliares de enfermagem "mais concordam que discordam" que o comportamento do enfermeiro é o estilo Grid de Liderança da gerência em equipe $(9,9)$, representando um estilo altamente desejável para os auxiliares. Quanto aos estilos 9,1; 1,9; 1,1 e 5,5, estas categorias não definem uma diferença significativa entre eles, visto que as alternativas apresentadas foram "mais concordo que discordo" ou "mais discordo que concordo" como caracterização do estilo de liderança do enfermeiro. 
Tabela 1 - Distribuição dos valores atribuídos pelos auxiliares de enfermagem (A) e técnicos de enfermagem (T) ao comportamento real do enfermeiro, segundo sujeitos da pesquisa e estilos Grid de Liderança, Diamantina, 2008

\begin{tabular}{|c|c|c|c|c|c|c|c|c|c|c|}
\hline \multirow{2}{*}{$\begin{array}{c}\text { Estilos Grid } \\
\text { Sujeitos }\end{array}$} & \multicolumn{2}{|c|}{9,1} & \multicolumn{2}{|c|}{1,9} & \multicolumn{2}{|c|}{1,1} & \multicolumn{2}{|c|}{5,5} & \multicolumn{2}{|c|}{9,9} \\
\hline & A & $\mathrm{T}$ & A & $\mathrm{T}$ & A & $\mathrm{T}$ & A & $\mathrm{T}$ & A & $\mathrm{T}$ \\
\hline 1 & 10 & 11 & 17 & 15 & 12 & 6 & 11 & 16 & 18 & 16 \\
\hline 2 & 14 & 14 & 13 & 14 & 11 & 11 & 14 & 17 & 11 & 9 \\
\hline 3 & 14 & 8 & 8 & 12 & 14 & 7 & 11 & 18 & 11 & 18 \\
\hline 4 & 15 & 9 & 6 & 13 & 11 & 6 & 7 & 14 & 5 & 18 \\
\hline 5 & 14 & 10 & 11 & 15 & 10 & 8 & 13 & 18 & 13 & 20 \\
\hline 6 & 11 & 7 & 14 & 19 & 11 & 5 & 15 & 20 & 14 & 20 \\
\hline 7 & 20 & 15 & 7 & 6 & 15 & 11 & 9 & 7 & 10 & 5 \\
\hline 8 & 9 & 15 & 14 & 6 & 9 & 11 & 20 & 7 & 20 & 5 \\
\hline 9 & 7 & 10 & 11 & 14 & 6 & 9 & 13 & 15 & 11 & 15 \\
\hline 10 & 5 & 10 & 13 & 14 & 4 & 9 & 16 & 14 & 20 & 18 \\
\hline 11 & 17 & 12 & 12 & 14 & 14 & 8 & 13 & 16 & 12 & 9 \\
\hline 12 & 12 & 5 & 10 & 9 & 5 & 3 & 8 & 9 & 15 & 12 \\
\hline 13 & 9 & 5 & 15 & 8 & 11 & 8 & 1 & 13 & 15 & 10 \\
\hline 14 & 17 & 7 & 5 & 10 & 16 & 12 & 6 & 9 & 9 & 9 \\
\hline 15 & 14 & 11 & 9 & 13 & 14 & 12 & 9 & 11 & 9 & 20 \\
\hline 16 & 7 & 11 & 17 & 14 & 5 & 12 & 14 & 14 & 20 & 19 \\
\hline 17 & 16 & 14 & 8 & 9 & 9 & 14 & 4 & 9 & 5 & 9 \\
\hline 18 & 17 & 5 & 16 & 14 & 13 & 5 & 16 & 11 & 15 & 20 \\
\hline 19 & 16 & 6 & 13 & 14 & 14 & 5 & 15 & 14 & 15 & 19 \\
\hline 20 & 12 & 12 & 16 & 16 & 5 & 5 & 12 & 12 & 19 & 19 \\
\hline 21 & 11 & 15 & 15 & 15 & 11 & 15 & 18 & 15 & 18 & 17 \\
\hline 22 & 18 & 16 & 16 & 16 & 12 & 6 & 16 & 16 & 16 & 17 \\
\hline Total & 285 & 228 & 266 & 280 & 232 & 188 & 273 & 297 & 301 & 334 \\
\hline $\bar{X}$ & 12,95 & 10,36 & 12,09 & 12,72 & 10,54 & 8,54 & 12,40 & 13,50 & 13,68 & 15,18 \\
\hline
\end{tabular}

Fonte: dados da pesquisa de campo

Quadro 2 - Apresentação da análise da variância das respostas informadas pelo Grupo 1, Diamantina, 2008

\begin{tabular}{|lcccc|}
\hline Fonte de Variação & GL & SQ & QM & Fc \\
Tratamento & 4 & 120,24 & 30,03 & $2,058^{\mathrm{NS}}$ \\
Bloco & 21 & 427,35 & 20,36 & \\
Erro & 84 & 1226,96 & 24,61 & \\
Total & & 1774,55 & & \\
\hline
\end{tabular}

Fonte: dados da pesquisa de campo.

Observações: $\mathrm{p}<0,01$; tratamento=estilo de liderança; bloco=22 auxiliares de enfermagem.

Legenda: GL: graus de liberdade; SQ: soma de quadrados; QM: quadrado médio e Fc: valor do teste F calculado.
De acordo com o Quadro 2, da análise de variância, o valor de Fc é menor que o valor tabelado de F com GL = 4; 120 e p < 0,01. Logo, o teste F é não significativo, não havendo diferença entre os estilos de liderança na análise do grupo dos auxiliares.

Para o teste $\mathrm{F}$ ser significativo, indicando diferenças entre os estilos de liderança, o valor de Fc deve ser maior que o valor de $\mathrm{F}$ tabelado, de acordo com os graus de liberdade do numerador (tratamento) e denominador (erro), bem como do nível de significância adotado (1\%).

Como não foi verificada a diferença entre os estilos de liderança, não há necessidade de se fazer o Teste de Duncan. Este teste só deve ser feito quando 
houver diferença entre os estilos.

Ainda, na Tabela 1, os técnicos de enfermagem "mais concordam que discordam" que o estilo de liderança real exercido pelo enfermeiro é o 9,9, ou seja, gerência em equipe. Os estilos de liderança 5,5; 1,9 e 9,1 foram classificados segundo os técnicos como "mais concordam que discordam" ou "mais discordam que concordam". Diferente dos auxiliares, os técnicos de enfermagem "mais discordam que concordam" que o estilo de liderança real exercido pelo enfermeiro é a gerência empobrecida $(1,1)$.

Quadro 3 - Apresentação da análise de variância das respostas informadas pelo Grupo 2, Diamantina, 2008

\begin{tabular}{|lcccc|}
\hline Fonte de Variação & GL & SQ & QM & Fc \\
Tratamento & 4 & 188,36 & 47,09 & $3,84^{*}$ \\
Bloco & 21 & 750,74 & 35,75 & \\
Erro & 84 & 1029,65 & 12,26 & \\
Total & & 1968,75 & & \\
\hline
\end{tabular}

Fonte: dados da pesquisa de campo.
Observações: $\mathrm{p}<0,01$; tratamento=estilo de liderança; bloco=22 técnicos de enfermagem.

Legenda: GL: graus de liberdade; SQ: soma de quadrados; QM: quadrado médio e Fc: valor do teste F calculado.

De acordo com o Quadro 3, o valor de Fc é maior que o valor tabelado de $\mathrm{F}$ com GL $=4$; 120 e p $<0,01$. Logo, o teste F é significativo, havendo diferença entre os estilos de liderança quando analisamos o grupo dos técnicos de enfermagem. Aplica-se, então, o Teste de Duncan ao Grupo 2.

Quadro 4a - Apresentação das médias dos estilos de liderança, Diamantina, 2008

\begin{tabular}{|cc|}
\hline Tratamento & Médias $\left(^{*}\right)$ \\
B & $41_{\mathrm{a}}$ \\
A & $38_{\mathrm{a}, \mathrm{b}}$ \\
$\mathrm{E}$ & $33_{\mathrm{a}, \mathrm{b}}$ \\
C & $25_{\mathrm{b}}$ \\
D & $24_{\mathrm{b}}$ \\
\hline
\end{tabular}

Quadro 4b - Apresentação das médias dos estilos de liderança e da diferença mínima significativa (DMS), de acordo com o Teste de Duncan, Diamantina, 2008

\begin{tabular}{|lcccccc|}
\hline Estilos & 1,1 & 9,1 & 1,9 & 5,5 & 9,9 & \\
Médias & $\bar{X}_{1}=8,5$ & $\bar{X}_{2}=10,3$ & $\bar{X}_{3}=12,7$ & $\bar{X}_{4}=13,5$ & $\bar{X}_{5}=15,18$ & DMS \\
8,5 & -- & 1,8 & 4,2 & 5 & $6,68^{*}$ & 6,53 \\
10,3 & & -- & 2,4 & 3,2 & 4,88 & 7,43 \\
12,7 & & -- & 0,8 & 2,48 & 7,96 \\
13,5 & & & -- & 1,68 & 8,34 \\
15,18 & & & & -- & \\
\hline
\end{tabular}

No Teste de Duncan, se a diferença encontrada entre as médias for maior que a DMS calculada, o teste será significativo. Pelos valores apresentados verifica-se que só houve diferença entre os estilos 9,9 (gerência em equipe) e 1,1 (gerência empobrecida).

Ao comparar os resultados encontrados com os mencionados na literatura ${ }^{(7)}$ é possível inferir que o estilo 9,9 e o estilo 1,1 são os referidos pelos técnicos de enfermagem. Contudo, há que se considerar a possibilidade de alguns falsos 9,9 que podem confundir os profissionais, dado que, por interesses distintos, muitas pessoas querem exteriorizar uma imagem de estilo 9,9 sem que, na verdade, assuma ações, comportamentos e atitudes que caracterizam esse valor, o que pressupõe um alto comprometimento tanto do líder quanto dos liderados para o desenvolvimento de metas e objetivos institucional e também em alcançar a expectativa destes profissionais, considerando sua criatividade, competência e participação em processos decisórios. Além disso, os dois estilos de liderança referidos pelos técnicos, 9,9 e 1,1 representam estilos de liderança opostos, sendo o primeiro (a gerência em equipe) desejável e o segundo (a gerência empobrecida) totalmente indesejável ${ }^{(9)}$.

Quanto à diferença significativa das proposições não encontrada no grupo dos auxiliares de enfermagem e encontrada nos técnicos de enfermagem, ela talvez se dê pela diferença na formação do auxiliar e técnico de enfermagem, em que estes possuem conteúdos de administração em enfermagem durante sua formação e, em algumas situações estes colaborarem com os enfermeiros em algumas funções gerenciais. 


\section{CONSIDERAÇÕES FINAIS}

O desenvolvimento deste estudo apontou que os auxiliares e os técnicos de enfermagem não diferenciaram o estilo de liderança 9,9 exercido pelo enfermeiro. Entretanto, os técnicos de enfermagem identificaram o estilo 9,9 e 1,1, sendo estes opostos: a gerência em equipe e a gerência empobrecida, o que pressupõe a coexistência de uma liderança que se aproxima mais de uma gestão flexível e uma liderança próxima a uma gestão baseada no Modelo Funcional, o que pode gerar visões distorcidas/contraditórias na assistência de enfermagem, não havendo coesão entre a equipe.

Acreditamos ser necessária a revisão do estilo de liderança exercido pelos enfermeiros na perspectiva de uma aproximação as exigências contemporâneas para a prestação da assistência a saúde, o que de certa forma, parece ser uma tendência quando os auxiliares e técnicos apontam o estilo 9,9 (a gerência em equipe) como um estilo de liderança do enfermeiro, mas que ao referenciar a gerência empobrecida que uma liderança que caracterize a interdependência por meio do comprometimento de todos com as metas e objetivos institucional e a valorização de decisões criativas tomadas entre líder e liderado em um clima de confiança e respeito ainda constitui um desafio ao enfermeiro.

Assim, cabe ao enfermeiro ampliar o conhecimento próprio e da equipe sobre a liderança, desde seu aspecto conceitual a sua aplicabilidade e benefícios, exercendo uma liderança que seja altamente orientada para as pessoas e para a produção, no intuito de garantir a satisfação profissional e a motivação humana no desenvolvimento do trabalho, com perspectivas a uma assistência de enfermagem diferenciada rumo a excelência do cuidado à clientela.

\section{REFERÊNCIAS}

1. Azevedo CS. Liderança e processos intersubjetivos em organizações públicas de saúde. Cienc Saúde Col. 2002;7(2):349-61.

2. Simões ALA, Fávero N. O desafio da liderança para o enfermeiro. Rev Latino-Am Enferm. 2003 out;11(5):567-73.

3. Lourenço MR, Trevizan MA. Líderes da enfermagem brasileira: sua visão sobre a temática da liderança e sua percepção a respeito da relação liderança e enfermagem. Rev Latino-Am Enferm. 2001 Mai;9(3):469-73.

4. Lourenço MR, Shinyashiki GT, Trevizan MA.
Gerenciamento e liderança: análise do conhecimento dos enfermeiros gerentes. Rev Latino-Am Enferm. 2005 Ago;13(4):469-73.

5. Galvão CM, Sawada NO, Castro AP, Corniani F. Liderança e comunicação: estratégias essenciais para o gerenciamento da assistência de enfermagem no contexto hospitalar. Rev Latino-Am Enferm. 2000 Out;8(5):34-43.

6. Santos I, Castro C. Estilos e dimensões da liderança: iniciativa e investigação no cotidiano do trabalho de enfermagem hospitalar. Texto Contexto Enferm. 2008 dez; 17(4): 734-742

7. Pereira MCA, Fávero N. A motivação no trabalho da equipe de enfermagem. Rev Latino-Am Enferm. 2001 Mai;9(4):7-12.

8. Gindri L, Medeiros HMF, Zamberlan C, Costernaro RGS. A percepção dos profissionais da equipe de enfermagem sobre o trabalho dos enfermeiros. Cogitare Enferm. 2005Abr;10(1):34-41.

9. Higa EFR, Trevizan MA. Os estilos de liderança idealizados pelos enfermeiros. Rev Latino-Am Enferm. 2005 Fev;13(1):59-64.

10. Trevisan MA. Liderança do enfermeiro: o ideal e o real no contexto hospitalar. São Paulo: Sarvier; 1993.

11. Blake R, Mouton JS. O novo grid gerencial. Trad. Lélio de Barros, $4^{\mathrm{a}}$ ed. São Paulo: Pioneira; 1987.

Cogitare Enferm 2009 Out/Dez; 14(4):628-37 


\section{ANEXO}

Instrumento Grid e Liderança em enfermagem: comportamento real ${ }^{(10)}$

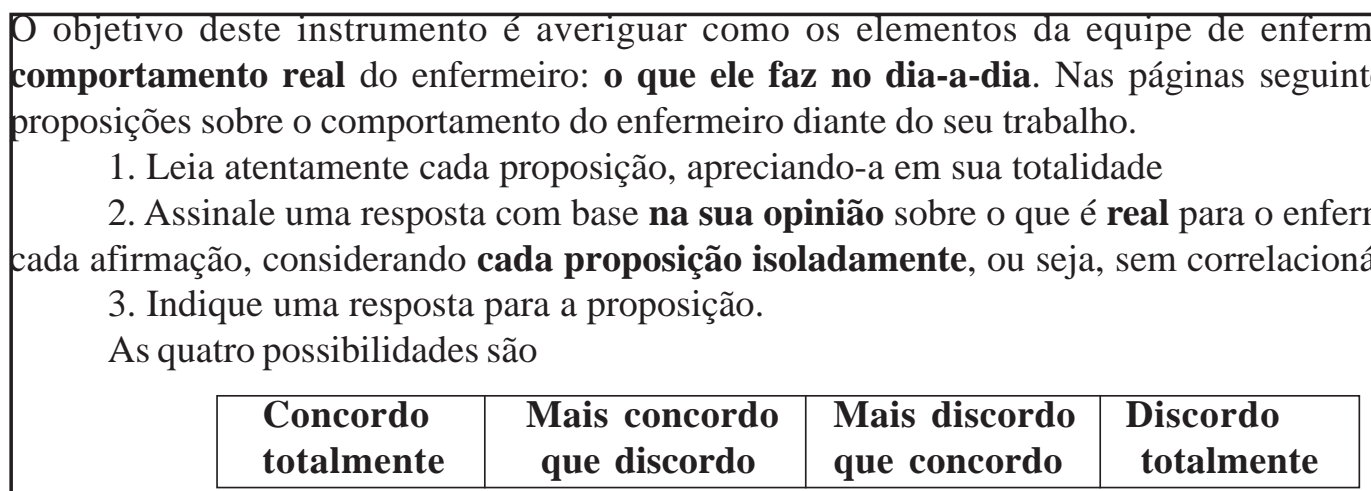

4. Não se preocupe se sua resposta está certa ou errada. O que interessa é a sua opinião

5. Cheque a indicação de apenas uma resposta para cada proposição. Suas respostas em nada influenciarão seu trabalho aqui no hospital

6. Você não deve colocar seu nome em nenhum lugar deste instrumento.

Preciso somente de sua identificação quanto a:

- Categoria profissional/ocupacional: ( ) 1.Técnico de Enfermagem ( ) 2.Auxiliar de Enfermagem

- Unidade de internação: (1) Médica

(2) Cirúrgica

(3) Centro de Terapia Intensiva

(4) Pronto Atendimento

- Idade:

- Sexo: ( ) 1.Masculino ( ) 2.Feminino

Muito obrigada pela colaboração!

\begin{tabular}{|c|c|c|c|c|}
\hline Proposição & $\begin{array}{l}\text { Concordo } \\
\text { plenamente }\end{array}$ & $\begin{array}{l}\text { Mais concordo } \\
\text { que discordo }\end{array}$ & $\begin{array}{l}\text { Mais discordo } \\
\text { que concordo }\end{array}$ & $\begin{array}{l}\text { Discordo } \\
\text { totalmente }\end{array}$ \\
\hline \multicolumn{5}{|l|}{$\begin{array}{l}\text { 1. O enfermeiro está sempre atento à opinião das pessoas, } \\
\text { mantendo uma posição intermediária entre as exigências do } \\
\text { serviço e as exigências das pessoas. }\end{array}$} \\
\hline \multicolumn{5}{|l|}{$\begin{array}{l}\text { 2. O enfermeiro demonstra pouca preocupação tanto com o } \\
\text { serviço quanto com as pessoas, esforça-se apenas o suficiente } \\
\text { para preservar seu emprego. }\end{array}$} \\
\hline \multicolumn{5}{|l|}{$\begin{array}{l}\text { 3. Se ocorre conflito ou tensão, o enfermeiro procura } \\
\text { conhecer os motivos, através da exposição aberta dos fatos } \\
\text { para, juntamente com os subordinados, solucionar as causas } \\
\text { do problema. }\end{array}$} \\
\hline \multicolumn{5}{|l|}{$\begin{array}{l}\text { 4. O enfermeiro faz todo o possível para que seus } \\
\text { subordinados se sintam satisfeitos com as condições do } \\
\text { trabalho e com o próprio chefe. }\end{array}$} \\
\hline \multicolumn{5}{|l|}{$\begin{array}{l}\text { 5. Para evitar o conflito o enfermeiro segue as normas e as } \\
\text { tradições do hospital ou se apóia na opinião da maioria; } \\
\text { quando ocorre o conflito, o enfermeiro busca soluções } \\
\text { conciliatórias para a situação. }\end{array}$} \\
\hline $\begin{array}{l}\text { 6. A atuação do enfermeiro propicia um clima de abertura, } \\
\text { franqueza e confiança. }\end{array}$ & & & & \\
\hline
\end{tabular}




\begin{tabular}{|c|c|c|c|c|}
\hline Proposição & $\begin{array}{l}\text { Concordo } \\
\text { plenamente }\end{array}$ & $\begin{array}{l}\text { Mais concordo } \\
\text { que discordo }\end{array}$ & $\begin{array}{l}\text { Mais discordo } \\
\text { que concordo }\end{array}$ & $\begin{array}{c}\text { Discordo } \\
\text { totalmente }\end{array}$ \\
\hline \multicolumn{5}{|l|}{$\begin{array}{l}\text { 7. Para o enfermeiro os resultados é que contam; assim, se } \\
\text { interessa muito pelos serviços e demonstra pouca } \\
\text { consideração com os subordinados, pressionando-os e } \\
\text { controlando-os para que sejam eficientes. }\end{array}$} \\
\hline \multicolumn{5}{|l|}{$\begin{array}{l}\text { 8. O relacionamento entre enfermeiro e subordinados se faz } \\
\text { através do manda e da obediência. }\end{array}$} \\
\hline \multicolumn{5}{|l|}{$\begin{array}{l}\text { 9. O padrão de comportamento do enfermeiro é } \\
\text { extremamente "democrático", com tendência para deixar os } \\
\text { subordinados muito à vontade no trabalho. }\end{array}$} \\
\hline \multicolumn{5}{|l|}{$\begin{array}{l}\text { 10. O enfermeiro dirige seus esforços visando unicamente } \\
\text { atender os objetivos do hospital. }\end{array}$} \\
\hline \multicolumn{5}{|l|}{$\begin{array}{l}\text { 11. Ao planejar e organizar o trabalho, o enfermeiro leva em } \\
\text { consideração as sugestões dos subordinados, e depois se } \\
\text { emprenha para que eles "comprem" as decisões tomadas. }\end{array}$} \\
\hline \multicolumn{5}{|l|}{$\begin{array}{l}\text { 12. O enfermeiro transmite as ordens superiores com a } \\
\text { máxima fidelidade, sem revelar suas opiniões sobre as } \\
\text { mesmas; o seu papel na transmissão destas ordens é de } \\
\text { "menino de recado". }\end{array}$} \\
\hline \multicolumn{5}{|l|}{$\begin{array}{l}\text { 13. O enfermeiro procura estar sempre atualizado na sua área } \\
\text { específica e na Enfermagem em geral, incentivando também os } \\
\text { subordinados para atualização. }\end{array}$} \\
\hline \multicolumn{5}{|l|}{$\begin{array}{l}\text { 14. O enfermeiro dá pouca importância aos objetivos do } \\
\text { serviço e atribui muita importância às necessidades, atitudes e } \\
\text { aos sentimentos dos subordinados. }\end{array}$} \\
\hline \multicolumn{5}{|l|}{$\begin{array}{l}\text { 15. Se ocorre o conflito, o enfermeiro procura manter-se } \\
\text { neutro, não se envolvendo. }\end{array}$} \\
\hline \multicolumn{5}{|l|}{$\begin{array}{l}\text { 16. O enfermeiro promove periodicamente encontros informais } \\
\text { com os subordinados, para rever o andamento do serviço, } \\
\text { ressaltando os pontos positivos, encorajando-os a superar } \\
\text { seus pontos fracos, e reduzindo a pressão sempre que } \\
\text { possível. }\end{array}$} \\
\hline \multicolumn{5}{|l|}{$\begin{array}{l}\text { 17. O comportamento do enfermeiro é participante e } \\
\text { centralizado no grupo. }\end{array}$} \\
\hline \multicolumn{5}{|l|}{$\begin{array}{l}\text { 18. O enfermeiro se empenha, sobretudo, em cultivar uma } \\
\text { atmosfera de calor humano no ambiente de trabalho. }\end{array}$} \\
\hline \multicolumn{5}{|l|}{$\begin{array}{l}\text { 19. O enfermeiro evita interferir nas tarefas dos subordinados } \\
\text { por não estar envolvido com o trabalho. }\end{array}$} \\
\hline \multicolumn{5}{|l|}{$\begin{array}{l}\text { 20. O enfermeiro atribui responsabilidade por tarefas } \\
\text { determinadas, dizendo a cada subordinado o que fazer, como, } \\
\text { quando, onde e com quem, e dá muita importância ao } \\
\text { desenvolvimento individual. }\end{array}$} \\
\hline \multicolumn{5}{|l|}{$\begin{array}{l}\text { 21. O enfermeiro procura alcançar integração entre serviço e } \\
\text { pessoas, para isso incentiva a participação e o compromisso } \\
\text { de todos na busca da melhor assistência de enfermagem. }\end{array}$} \\
\hline $\begin{array}{l}\text { 22. As avaliações de desempenho são executadas de maneira } \\
\text { superficial pelo enfermeiro. }\end{array}$ & & & & \\
\hline
\end{tabular}

Continua 


\begin{tabular}{|l|l|l|l|l|}
\hline Proposição & $\begin{array}{c}\text { Concordo } \\
\text { plenamente }\end{array}$ & $\begin{array}{c}\text { Mais concordo } \\
\text { que discordo }\end{array}$ & $\begin{array}{c}\text { Mais discordo } \\
\text { que concordo }\end{array}$ & $\begin{array}{c}\text { Discordo } \\
\text { totalmente }\end{array}$ \\
\hline $\begin{array}{l}\text { 23. Quando ocorrer conflito o enfermeiro usa sua autoridade } \\
\text { para removê-lo, fazendo prevalecer sua própria decisão }\end{array}$ & & & \\
\hline $\begin{array}{l}\text { 24. O enfermeiro mantém as portas sempre abertas para seus } \\
\text { subordinados, procurar atender a todas suas solicitações, e } \\
\text { congratula-se efusivamente com eles pelo andamento dos } \\
\text { trabalhos, evitando críticas que possam constrangê-los }\end{array}$ & & & \\
\hline $\begin{array}{l}\text { 25. O enfermeiro se preocupa em garantir uma aceitável } \\
\text { assistência de enfermagem, sem incomodar demais seus } \\
\text { subordinados. }\end{array}$ & & & & \\
\hline
\end{tabular}

